\title{
PUBLISHED AS
}

A. Léger, N. R. Calderon, R. Charvet, W. Dufour, C. Bacciarini, L. Weber, A. Mortensen' "Capillarity in pressure infiltration: improvements in characterization for high-temperature systems", Journal of Materials Science, 47 (24) pp 8419-8430 (2012), doi:10.1007/s10853-012-6645-2.

\section{Capillarity in pressure infiltration: improvements in characterization for high-temperature systems}

\author{
A. Léger ${ }^{\mathrm{a}, *}$, N. R. Calderon ${ }^{\mathrm{b}}$, R. Charvet ${ }^{\mathrm{a}}, \mathrm{W}$. Dufour $^{\mathrm{a}}, \mathrm{C}$. Bacciarini $^{\mathrm{c}}, \mathrm{L}$. \\ Weber $^{a}$, A. Mortensen ${ }^{a}$ \\ a Laboratory of Mechanical Metallurgy, Institute of Materials, École Polytechnique Fédérale de Lausanne \\ (EPFL), Station 12, CH-1015 Lausanne, Switzerland \\ b Now with: Philip Morris Products S.A., CH-2000 Neuchâtel, Switzerland \\ c Now with: Innovation Cells, Constellium, CH-1015 Lausanne, Switzerland \\ *Corresponding author <alain.leger@epfl.ch>
}

\begin{abstract}
In the pressure infiltration of metal-matrix composites, molten metal is injected under external pressure into a porous preform of the reinforcing material. Equilibrium capillary parameters characterizing wetting for this process are summarized in plots of metal saturation versus applied pressure, also known as drainage curves. Such curves can be measured in our laboratory during a single experiment with an infiltration apparatus designed to track the rate of metal penetration into porous preforms under conditions characteristic of metal matrix composite processing (temperatures in excess of $1000{ }^{\circ} \mathrm{C}$ and pressures in the order of $10 \mathrm{MPa}$ ). For such measurements to be valid, infiltration of the preform with molten metal must be mechanically quasi-static, i.e., the metal must flow at a rate sufficiently low for the metal pressure to be essentially uniform across the preform at all times. We examine this requirement quantitatively, using a finite-difference model that simulates the unsaturated unidirectional ingress of molten metal into a ceramic particle preform of finite width. We furthermore present improvements in the experimental apparatus developed in our laboratory to measure the entire drainage curve in a single experiment. We confront numerical results with new experimental data for the copper/alumina system, to show (i) that pressurization rates sufficiently low for quasistatic infiltration can be produced with this apparatus, and (ii) that taking the relative permeability equal to the saturation yields better agreement with experiment than does the expression originally proposed by Brooks and Corey.
\end{abstract}




\section{Introduction}

Capillary forces are of primary importance in materials processing and this is particularly true in metal-matrix composite (MMC) production by liquid metal infiltration. In this process, molten metal fills a porous preform of the reinforcement phase, after which it is solidified within the preform pores, resulting in a composite material. In liquid metal infiltration, capillary forces are typically much higher than gravitational forces. Furthermore, most metals wet poorly most practically interesting reinforcements. Therefore, spontaneous infiltration is rarely observed: external force must generally be applied to drive the molten metal into the preform. As result, most infiltrated metal matrix composites are produced by pressure infiltration [1-10].

The basic capillary parameter governing infiltration is the work of immersion $W_{\mathrm{i}}$; this represents the work necessary to wet a unit area of initially dry reinforcement:

$$
W_{\mathrm{i}}=\sigma_{\mathrm{SL}}-\sigma_{\mathrm{SV}}
$$

where $\sigma_{\mathrm{SV}}$ and $\sigma_{\mathrm{SL}}$ are the solid-vapor and solid-liquid surface energies, respectively. The work of immersion, $W_{\mathrm{i}}$, is related to the contact angle, $\theta$, of the metal on the ceramic by the Young equation:

$$
\cos \theta=\frac{\sigma_{\mathrm{SV}}-\sigma_{\mathrm{SL}}}{\sigma_{\mathrm{LV}}}=-\frac{W_{\mathrm{i}}}{\sigma_{\mathrm{LV}}}
$$

expressing capillary equilibrium at a stationary triple line along a non-deforming solid; here $\sigma_{\mathrm{LV}}$ is the liquid metal surface tension and $\theta>90^{\circ}$ signals a non-wetting system.

The study of wetting, also at elevated temperature, is nearly always associated with the sessile drop experiment. This consists of measuring the equilibrium contact angle $\theta$ of a liquid droplet resting on a planar substrate, $\theta$ being given by the Young equation (Eq. 2) [11-13]. At elevated temperature, the sessile drop experiment is delicate: challenges that come with high temperatures and the need for tight atmospheric control lead to frequent discrepancies in reported $\theta$ values for metal/ceramic systems $[12,14]$. With infiltration, furthermore, there is a second difficulty with the technique: a single equilibrium contact angle value is insufficient to fully characterize wetting of a preform by flowing metal, for two reasons. The first is that wetting in infiltration is dynamic: local capillary equilibrium is generally not reached at a moving liquid metal front, which furthermore in pressure infiltration corresponds to forced, rather than spontaneous, wetting. The second has to do with geometry: the invasion by fluid of a complex 3-D labyrinth of irregularly shaped open pores differs from wetting by a drop of a flat surface: molten metals, as most other fluids, invade porous preforms over a range of pressures. This range of pressures, in turn, is dictated by the geometry of open pores within the ceramic preform and by the intensity of capillary forces that equilibrate at a triple line, the exact location of which is a priori unknown. Specifically, with non-wetting fluids, larger pores are invaded first, as these present the smallest resistance to flow, as regards both capillary and viscous forces. Finer pores are only infiltrated later, when the pressure in the metal has reached higher values [2, 5, 8, 15-18].

The importance of capillary forces in infiltration processing of composite materials, and particularly in the production of metal matrix composites (given the high value of the liquid surface tension in metals [8]) has motivated a sustained research effort aiming to quantify and hence measure their value. In essence, two basic approaches have been used to characterize wetting of porous preforms by molten metals during infiltration; these are contrasted and compared in more detail in Refs. [19-20]. 
The first approach explores infiltration under strongly driven flow. Here, experiments are designed to address the fact that wetting in infiltration is dynamic; the capillary number $\mathrm{Ca}$ (defined as viscosity times velocity over surface tension) is therefore high. The simplest practical method is to infiltrate unidirectionally a series of samples, varying the pressure while keeping the time fixed. Alternatively, some teams have conducted forced unidirectional infiltration experiments using set-ups that can sense, in time, the progression of metal into the porous solid body; the ratio of infiltration length squared to time is then measured for a series of (constant) applied pressures. Provided infiltration takes place with a sufficiently well-defined planar infiltration front (something that is called "slug flow"), one can back-calculate by plotting the position of the infiltration front, $h$, squared versus time, the capillary pressure drop $\Delta P_{\gamma}$ between the atmosphere and the metal across the infiltration front. From this, making the assumption that infiltration takes place with negligible irreversible energy loss, the work of immersion $W_{\mathrm{i}}$ (and thus the contact angle $\theta$ according to Eq. 2) can be deduced according to:

$$
\Delta P_{\gamma}=\frac{V_{\mathrm{r}} A_{\mathrm{v}} W_{\mathrm{i}}}{1-V_{\mathrm{r}}}=-\frac{V_{\mathrm{r}} A_{\mathrm{v}} \sigma_{\mathrm{LV}} \cos \theta}{1-V_{\mathrm{r}}}
$$

where $V_{\mathrm{r}}$ is the volume fraction reinforcement in the preform and $A_{\mathrm{v}}$ is the reinforcement surface area per unit volume of the reinforcement. Such experiments have been conducted by several laboratories and, when properly conducted and interpreted, give reliable and consistent results; reviews are given in Refs. [2, 5, 9].

The other approach addresses the geometrical complexity of porous medium infiltration. Here, experiments are designed to produce the opposite extreme, of very slow infiltration (i.e., low $C$ a); those are conditions of interest in the present work.

Approaches aimed at tackling the geometrically complex and gradual nature of infiltration as it obtains under capillary and mechanical equilibration of the fluid in the pores come mainly from branches of engineering other than materials science: soil science and petroleum engineering notably $[15-16,21]$. Characterization of pore filling by non-wetting fluids in these disciplines is generally conducted by measurement of drainage curves; these are plots of the volume fraction of non-wetting fluid in the porous medium versus the pressure difference $\Delta P$ between the fluid and the atmosphere in the pores. Each point along a drainage curve is measured after equilibration, meaning after cessation of fluid flow - in fact, this is what is done at room temperature in a mercury porosimeter. This approach has been extended to high temperatures (e.g., $1000{ }^{\circ} \mathrm{C}$ ) and high applied pressures (e.g., $10 \mathrm{MPa}$ ) characteristic of ceramic-metal systems [17-18, 22-26]. One method is to infiltrate a series of preforms at different pressures for times long enough that the metal is uniformly distributed throughout the sample, and then measure metallographically or by densitometry the volume fraction of metal, and then plot this versus pressure to deduce discrete points along the drainage curve $[17,22,24]$. This discontinuous method is time-consuming and labour-intensive. A far more efficient way is to track dynamically the volume of metal that has entered the preform (as is practiced in a mercury porosimeter).

The two approaches described above are linked if infiltration of the porous solid preform takes place, in both cases (meaning both at high and at low $C a$ ), with negligible irreversible energy loss at the triple line. Then, the drainage curve integrated with respect to saturation of the nonwetting fluid (defined below in Eq. 7), $W$, should equal the work of immersion, $W_{\mathrm{i}}$, multiplied by the total area of preform/infiltrant interface created per unit volume of reinforcement, namely :

$$
W=\int_{0}^{1} \Delta P d S=W_{\mathrm{i}} A_{\mathrm{V}} V_{\mathrm{r}}=-\sigma_{\mathrm{LV}} \cos \theta A_{\mathrm{V}} V_{\mathrm{r}}=\left(1-V_{\mathrm{r}}\right) \Delta P_{\gamma}
$$

where $\Delta P$ is the pressure difference between the fluid and the atmosphere in the pores at saturation $S$ in the preform after equilibration. 
Our laboratory has developed a gas-pressure infiltration apparatus with adequate sensing equipment to provide the entire drainage curve in a single experiment with high-melting point metals such as copper. The system, in its first version, comprised a linear variable differential transformer (LVDT) that was connected (via an alumina tube) to a graphite plunger floating atop the liquid metal. When infiltration starts, the plunger moves with the metal, such that by measuring its displacement, the saturation can be directly deduced as a function of time and pressure. Further details on this infiltration apparatus can be found elsewhere [24]. This technique gave reliable results that matched, at room-temperature, those of a mercury porosimeter and, at elevated temperature, those of the discontinuous method for copper infiltrating particulate alumina preforms [24].

We have recently improved this technique, with a goal to ensure quantitatively that infiltration is indeed quasi-static, meaning that, within acceptable precision, the metal volume fraction is uniform across the preform at all times (or in other words that viscous pressure gradients within the fluid are negligible). We present here these improvements, together with a quantitative analysis of the process based on a finite-difference simulation of isothermal infiltration of a rigid preform under an arbitrary increasing-pressure function. Our aim is to define requirements for quasi-static infiltration and to show that these can be met experimentally.

\section{Theory}

To quantify requirements for quasi-static isothermal infiltration, the internal distribution of metal saturation within the preform must be predicted as a function of time knowing the infiltration process pressurization cycle. On this basis, then, pressurization cycles can be designed such that saturation gradients within the sample are sufficiently low at the time of measurement for the resulting drainage curve to be sufficiently accurate.

To this end, a finite difference model was developed to simulate the unsaturated ingress of molten metal into a ceramic particle preform. The physics of the process are similar to drainage phenomena encountered in soil mechanics or reservoir engineering; governing laws can be found in Refs. [2, 5, 15-17, 23, 27-28].

The model addresses the unidirectional isothermal infiltration of an evacuated nondeformable inert preform by a non-wetting fluid. Flow of the fluid is governed by continuity (Eq. 5) and Darcy's law (Eq. 6).

$$
\begin{aligned}
& \frac{\partial V_{\mathrm{m}}}{\partial t}=-\operatorname{div}\left(v_{0}\right) \\
& v_{0}=-\frac{k_{\mathrm{e}}}{\mu} \operatorname{grad}(P)
\end{aligned}
$$

where $V_{\mathrm{m}}$ is the local volume fraction of metal, $v_{0}$ is the vectorial superficial velocity, $\mu$ is the viscosity of the liquid metal, $k_{\mathrm{e}}$ is the porous medium permeability at the relevant local metal saturation, and $P$ is the local pressure (the effect of gravity is generally negligible in metal infiltration [5]). It has been shown [17-18, 23, 29-33] that quasi-static drainage curves measured at high temperature (discontinuously or continuously) with molten metals and ceramic preforms follow a law known as the Brooks-Corey correlation [16, 28, 34-35]. This links the capillary pressure $P$ and drainage saturation, according to :

$$
S \equiv \frac{V_{\mathrm{m}}}{1-V_{\mathrm{r}}}=1-\left(\frac{P_{\mathrm{b}}}{P}\right)^{\lambda}
$$


where $S$ is the saturation in non-wetting fluid (here the metal), $V_{\mathrm{r}}$ is the local volume fraction of reinforcement and $P$ is the pressure difference between the non-wetting fluid (the metal) and the wetting fluid (the latter being nil since the preforms are initially evacuated). Two parameters underlie the correlation, namely $P_{\mathrm{b}}$, the "bubbling pressure" (related to the size of the largest pore forming a continuous network) and $\lambda$, a "pore size distribution index", which measures the distribution of sizes of the flow channels within the porous medium [16, 27-28, 34].

In unsaturated flow, the local "effective" permeability $k_{\mathrm{e}}$ is a function of $V_{\mathrm{m}}$. This can be expressed by taking $k_{\mathrm{e}}$ as the product of the permeability of the fully saturated medium $k_{\mathrm{s}}$ reduced by an $S$-dependent factor less than unity, known as the relative permeability $k_{\mathrm{r}}$ :

$$
k_{\mathrm{e}}=k_{\mathrm{r}} k_{\mathrm{s}}
$$

The saturated permeability $k_{\mathrm{s}}$, characteristic of the preform, can be directly measured, for example by flowing water through the preform (e.g., [36]).

Brooks and Corey also proposed expressions for the relative permeability, $k_{\mathrm{r}}$, the equation being different for a wetting or a non-wetting fluid [34]. For a non-wetting fluid, the expression proposed by Brooks and Corey reads:

$$
k_{\mathrm{r}}=\left[1-\left(\frac{P_{\mathrm{b}}}{P}\right)^{\lambda}\right]^{2}\left[1-\left(\frac{P_{\mathrm{b}}}{P}\right)^{2+\lambda}\right]
$$

Dopler et al. found, on comparing predictions from a model of pressure infiltration with data for aluminum infiltrating preforms of chopped short alumina fibers, that $k_{\mathrm{r}}$ was more adequately predicted by the following simple expression [23]:

$$
k_{\mathrm{r}}=S
$$

The latter expression, which in Mualem's review corresponds to a Kozeny-type expression of exponent of unity [37], in fact corresponds to a very simple view of the porous medium, namely one in which the medium is separated into parallel regions much larger than the characteristic pore size, that are either fully filled with liquid or empty. These two expressions for $k_{\mathrm{r}}$ are compared in Fig. $1:$ the Brooks and Corey expression supposes a much stronger reduction in permeability with decreasing $S$ than does the linear relation in Eq. (10).

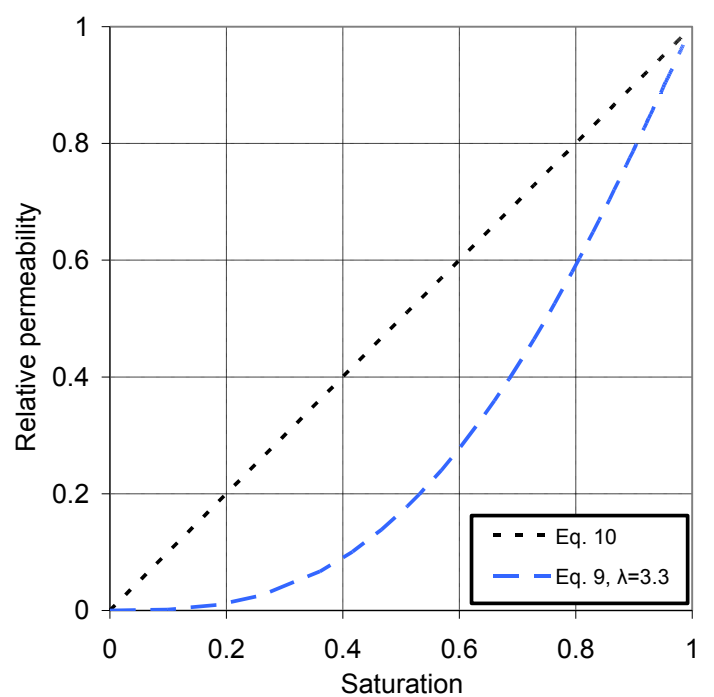

Fig. 1. Relative permeability $k_{\mathrm{r}}$ as a function of saturation $S$ for the expressions given by Eq. 9 and Eq. 10 . 
Eq. 5 can be rewritten using Eq. 7; after incorporation in Eqs. (5) and (6) combined and rewritten in terms of the metal saturation $S$ :

$$
\frac{\partial V_{\mathrm{m}}}{\partial t}=\frac{\partial\left(S\left(1-V_{\mathrm{r}}\right)\right)}{\partial t}=\left(1-V_{\mathrm{r}}\right) \frac{\partial S}{\partial t}
$$

One then obtains a single differential equation, which writes for 1-D linear infiltration along the $x$ direction:

$$
\frac{\partial S}{\partial t}=\frac{k_{\mathrm{s}}}{\mu\left(1-V_{\mathrm{r}}\right)} \frac{\partial}{\partial x}\left(k_{\mathrm{r}} \frac{\partial P}{\partial S} \frac{\partial S}{\partial x}\right)=\frac{k_{\mathrm{s}}}{\mu\left(1-V_{\mathrm{r}}\right)} \frac{\partial}{\partial x}\left(f(S) \frac{\partial S}{\partial x}\right)
$$

and for radial infiltration in cylindrical symmetry

$$
\frac{\partial S}{\partial t}=\frac{k_{\mathrm{s}}}{\mu\left(1-V_{\mathrm{r}}\right)}\left[\frac{\partial}{\partial r}\left(f(S) \frac{\partial S}{\partial r}\right)+\frac{f(S)}{r} \frac{\partial S}{\partial r}\right]
$$

where the term $f(S)=k_{\mathrm{r}} \frac{\partial P}{\partial S}$.

This non-linear equation was solved numerically using the finite-difference method, for an initially dry preform of finite width $L$, immersed on both sides $(x=0$ and $x=L)$ in liquid metal subjected to a pressure $P$ that is a prescribed function of time. The equation was solved using an explicit Euler method for time discretization and a Taylor development to first order for the saturation $S$ (in Visual ${ }^{\circledR}$ ) by incorporating respectively Eq. 7, 8 and 9 (or 10) into the code. A slight challenge in designing the code was to devise a suitable method of dealing with the (abrupt) moving infiltration front implied by the Brooks and Corey correlation: precisely at the infiltration front, where $P$ equals $P_{\mathrm{b}}$, the saturation $S$ and hence $f(S)$ fall to zero. To avoid artificial flow blockage at that point in the calculation, in the explicit finite-difference scheme, $f(S)$ was calculated using the average value of $S$ taken for the two neighbouring nodes. This gives $f(S)$ a finite (albeit at first very small) value at every node situated between the flow entrance point and a distance equal to the distance discretization step $\Delta x$ times the number of iterations in time of the simulation. This did away with a limitation that has often motivated use of the less physically correct but computationally more convenient van Genuchten correlation [38].

\section{Experimental methods}

\section{A. Materials}

Alumina particles were similar to those used in $\operatorname{Ref}[18,24]$. These were of two types, namely (i) angular $\alpha-\mathrm{Al}_{2} \mathrm{O}_{3} \mathrm{~F} 1000$ purchased from Treibacher Schleifmittel (Laufenburg, Germany), and polygonal Sumicorundum AA-5, produced by Sumitomo Chemicals Co., Ltd. (Osaka, Japan). Both F1000 and AA-5 alumina powders have an average diameter of around $5 \mu \mathrm{m}$. Preforms from the angular F1000 powder were prepared in two different ways: by tapping and vibrating the powder directly into its crucible up to a height of $15 \mathrm{~mm}$, or alternatively by cold isostatic pressing (CIP) at $150 \mathrm{MPa}$. The obtained CIP preforms were cylindrical, about $14 \mathrm{~mm}$ in diameter and $15 \mathrm{~mm}$ in height.

The volume fraction of reinforcement ceramic $V_{\mathrm{r}}$ in the preforms (and hence in the composites) was measured by densitometry using a Sartorius MC $210 \mathrm{P}$ microbalance of sensitivity $\pm 10 \mu \mathrm{g}$ assuming full infiltration of the composites produced from the preforms. The two packing methods yield volume fractions ceramic $V_{\mathrm{r}}=0.48$ and 0.53 , respectively. The volume fraction ceramic in AA-5 powder preforms pressed by CIP at the same pressure is $V_{\mathrm{r}}$ $=0.63$. 
The saturated permeability $k_{\mathrm{s}}$ of water through tapped $\mathrm{F} 1000 \mathrm{Al}_{2} \mathrm{O}_{3}$ preforms was measured experimentally in a polymethyl methacrylate (PMMA) tube equipped with a differential pressure gage and filters retaining the particles at both ends. A series of measurements at pressures in the range of 1 to 5 bar were conducted by recording the mass of the permeating liquid over 10 minutes. The permeability was deduced from the measured flow rate using Darcy's law as $k_{\mathrm{s}}=7$ $\cdot 10^{-15} \mathrm{~m}^{2}$ for the tapped $\mathrm{F} 1000 \mathrm{Al}_{2} \mathrm{O}_{3}$ preforms, the value varying only little with applied pressure up to 5 bar. Cold isostatically pressed preforms of the same powder have a somewhat higher density; their permeability was estimated by correcting the value measured with tapped preforms for the difference in porosity using the Blake-Kozeny relation [39]. This gave $k_{\mathrm{s}}=4.2 \cdot 10^{-15} \mathrm{~m}^{2}$ for the CIPped $\mathrm{F} 1000 \mathrm{Al}_{2} \mathrm{O}_{3}$ preforms. Electrolytic tough pitch (ETP) copper was used as the molten infiltrating matrix. At $1100{ }^{\circ} \mathrm{C}$, its viscosity is $4.3 \cdot 10^{-3} \mathrm{~Pa} \cdot \mathrm{s}$, at $1150{ }^{\circ} \mathrm{C}: 3.9 \cdot 10^{-3} \mathrm{~Pa} \cdot \mathrm{s}$ and at $1200{ }^{\circ} \mathrm{C}: 3.6 \cdot 10^{-3} \mathrm{~Pa} \cdot \mathrm{s}[40]$.

\section{B. Gas-pressure infiltration}

The gas-pressure infiltration apparatus is a cold-wall device in which heating is provided by an induction coil surrounding a graphite susceptor. A general description of the apparatus is given in [24]. Infiltration is typically conducted in alumina or quartz crucibles; within these a preform is placed below a slug of metal. Once a primary vacuum is established within the apparatus under continued pumping, the metal melts and the preform is sealed from the surrounding atmosphere. After 15 minutes of thermal equilibration at the infiltration temperature, argon pressure is applied to drive the molten metal into the evacuated preform. Compared to the earlier version of the apparatus described in Ref. [24], the following modifications were made:

- An automated system of pressure control composed of a set of electrovalves that regulate the gas inlet and outlet up to $20 \mathrm{MPa}$ with $5 \mathrm{kPa}$ precision. This in turn allows for (i) improved precision in control of pressure and (ii) much lower constant pressurization rates.

I - A new copper induction coil and graphite susceptor were produced and installed, and the induction heating unit was changed, with an eye to improve heating of the crucible. This was needed to maintain isothermal conditions during prolonged infiltration experiments - in turn enabling the exploration of much lower pressurization rates.

- Thermal insulation structures within the apparatus were changed to reduce heat losses and to protect delicate measurement devices from heating, again with a goal to enable experiments of much longer duration.

- An entirely new optical system was built to track motion of the floater, this in turn measuring the metal volume entering into the porous body during infiltration. The main reason for changing the tracking device was that the greater power used in heating for prolonged experiments resulted in a stronger electromagnetic field, this in turn perturbing the signal given by the former, linear variable differential transformer (LVDT) based, system. This was a difficult problem to solve since all commercial measurement devices contain electronic parts, and hence cannot be used in the vicinity of the induction-generated electromagnetic field. A new optical custom-made system was thus conceived and developed, by replacing the adapter formerly containing the LVDT with an adapter featuring two (pressure and vacuum resistant) custom-made windows, one for a light source and the other for capture by a digital camera of a series of white stripes lining the rod that connects the rotative and vibrative systems to the graphite plunger. This in turn allows to track (out of the magnetic field) the movement of the liquid metal level. The old and the new set-ups are compared in Fig. 2. A dedicated LabView software was also developed to compute the metal level position by image analysis. The resolution of the system is in the order of several micrometres while the precision (after calibration) is around $40-50 \mu \mathrm{m}$ for a $6 \mathrm{~mm}$ displacement of the graphite plunger. 


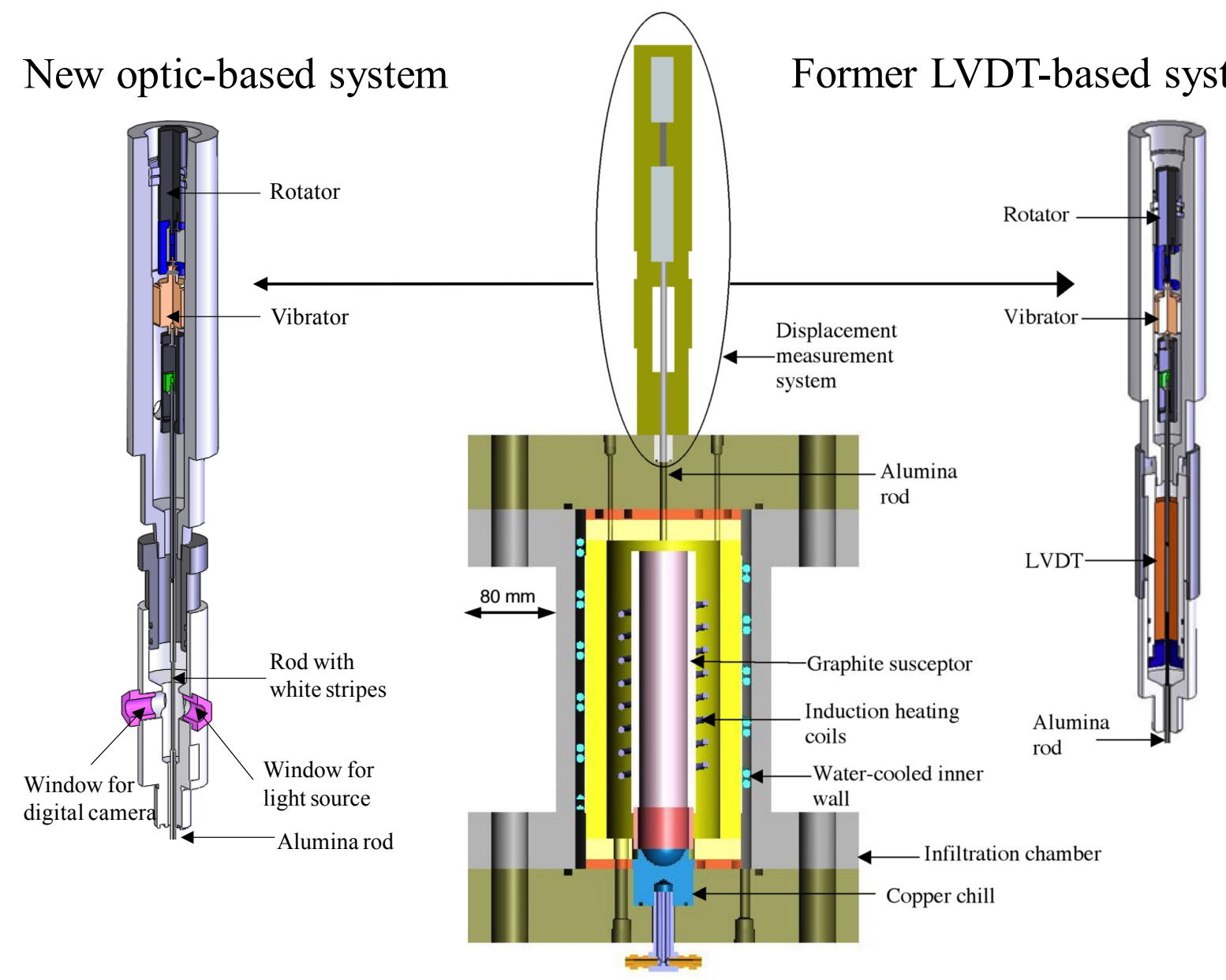

Fig. 2. Schematic of metal level tracking device fixed on top of infiltration apparatus, showing main differences between the former LVDT-based system (right) and the new optic-based system (left).

\section{Infiltration experiments}

A first set of drainage experiments was performed to test the precision of the infiltration device. These experiments were conducted with a nominally constant pressurization rate during the entire infiltration run. This was performed for (i) $\mathrm{Al}_{2} \mathrm{O}_{3}(\mathrm{AA}-5) / \mathrm{Cu}$ at $0.005 \mathrm{MPa} / \mathrm{s}$ and (ii) $\mathrm{Al}_{2} \mathrm{O}_{3}(\mathrm{~F} 1000) / \mathrm{Cu}$ at either $0.04 \mathrm{MPa} / \mathrm{s}$ or $0.005 \mathrm{MPa} / \mathrm{s}$, in both cases at $1200{ }^{\circ} \mathrm{C}$. Experiments with polygonal (AA-5) alumina were done for comparison of the new system with earlier results gathered using the older apparatus.

A second set of drainage experiments were carried out on F1000 alumina preforms only. These were infiltrated with copper under stepwise increasing pressure, holding each pressure level for a time long enough that the displacement of the graphite plunger reached a plateau, or in other words, for the saturation to stabilize. Discrete points in the saturation curve were thus obtained, as in discontinuous experiments - but with the difference that each point did not necessitate the preparation and metallographic characterization of a separate sample. Two different types of preforms of $\mathrm{F} 1000 \mathrm{Al}_{2} \mathrm{O}_{3}$ were studied in this manner: one preform produced by tapping and vibrating was infiltrated at $1100{ }^{\circ} \mathrm{C}$ while another preform made by CIP was infiltrated at $1150{ }^{\circ} \mathrm{C}$. Note that these two configurations differ in the infiltration path: tapped preforms fill the bottom of the crucible; hence these are mainly infiltrated from the top preform surface downwards. CIPped preforms rest on their edges upon the (rounded) bottom of the crucibles; hence these are surrounded by molten metal and infiltrated from all sides. 


\section{Experimental results}

\section{A. Continuous drainage curves}

Figure 3 compares the measured evolution of pressure and temperature versus time during the infiltration of alumina with copper before (a) and after (b) the improvements that were brought to the apparatus.
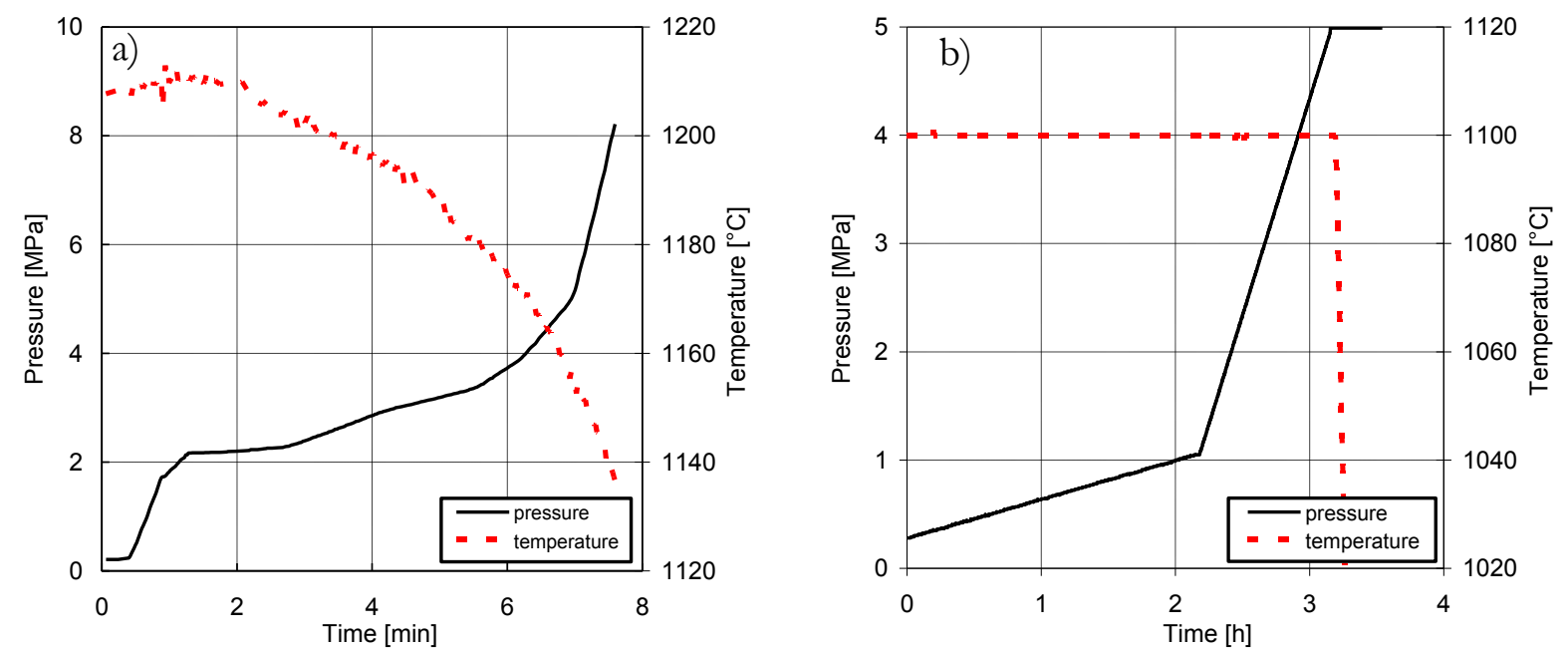

Fig. 3. Typical variation of pressure and temperature as a function of time during two experiments (conducted with the alumina-copper system) for (a) the former [41] and (b) the new set up, in which two different pressurization rates $\left(10^{-4} \mathrm{MPa} / \mathrm{s}\right.$ followed by $\left.1.1 \cdot 10^{-3} \mathrm{MPa} / \mathrm{s}\right)$ were maintained.

A continuous drainage curve for $\mathrm{Al}_{2} \mathrm{O}_{3}(\mathrm{AA}-5) / \mathrm{Cu}(0.005 \mathrm{MPa} / \mathrm{s})$ at $1200{ }^{\circ} \mathrm{C}$ is displayed in Figure $4 \mathrm{a}$ and compared with results obtained in earlier work [41] for the same system (at 0.04 $\mathrm{MPa} / \mathrm{s}$ ). Figure 4b compares drainage curves for $\mathrm{Al}_{2} \mathrm{O}_{3}(\mathrm{~F} 1000) / \mathrm{Cu}$, both obtained with the new apparatus but at two different pressurization rates $\left(\mathrm{d} P_{\text {applied }} / \mathrm{d} t=0.04 \mathrm{MPa} / \mathrm{s}\right.$ and $\left.0.005 \mathrm{MPa} / \mathrm{s}\right)$, also at $1200{ }^{\circ} \mathrm{C}$.
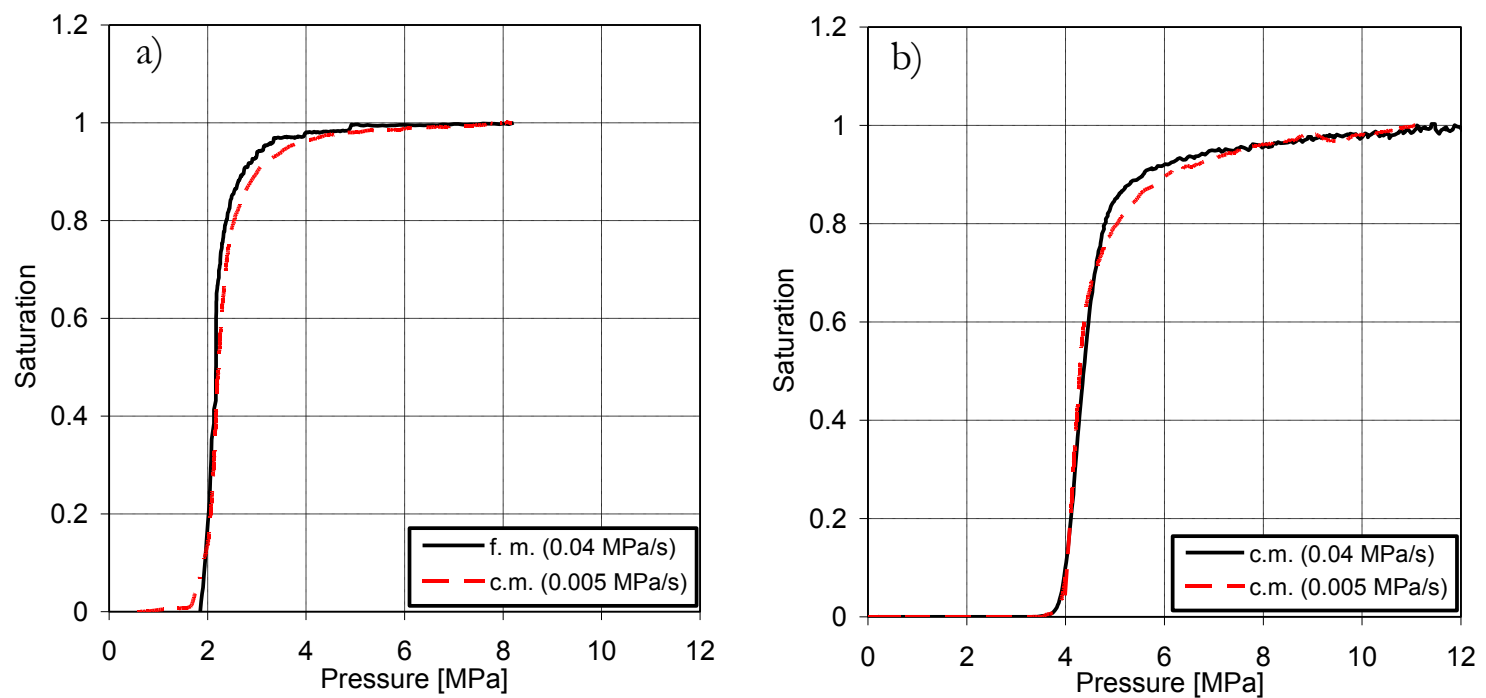

Fig. 4. Drainage curves at $1200{ }^{\circ} \mathrm{C}$ for a) polygonal AA-5 $\mathrm{Al}_{2} \mathrm{O}_{3} / \mathrm{Cu}\left(V_{r}=0.63\right)$ and b) angular $\mathrm{F} 1000 \mathrm{Al}_{2} \mathrm{O}_{3} / \mathrm{Cu}\left(V_{r}\right.$ $=0.53)$ at $1200{ }^{\circ} \mathrm{C}$, comparing data for two pressurization rate values, $\mathrm{d} P_{\text {applied }} / \mathrm{d} t=0.04$ or $0.005 \mathrm{MPa} / \mathrm{s}$, including data from the present apparatus (c.m: current method) and former work (f. m., from Ref. [18]). The preforms were produced by cold isostatic pressing at $150 \mathrm{MPa}$ and $S=1$ was assumed at $8 \mathrm{MPa}$ for the polygonal AA-5 $\mathrm{Al}_{2} \mathrm{O}_{3} / \mathrm{Cu}$ and at $11 \mathrm{MPa}$ for angular $\mathrm{F} 1000 \mathrm{Al}_{2} \mathrm{O}_{3} / \mathrm{Cu}$. 
B. Discontinuous drainage curves.

Figure 5 presents discrete points measured after step-wise pressure increases for (i) a preform made of $\mathrm{Al}_{2} \mathrm{O}_{3}$ (F1000) by tapping and vibrating $\left(V_{r}=0.48\right)$ and (ii) a preform of the same powder made by cold isostatic pressing at $150 \mathrm{MPa}\left(V_{r}=0.53\right)$. Each curve is fitted with the Brooks and Corey (BC) correlation on the figure (see below).

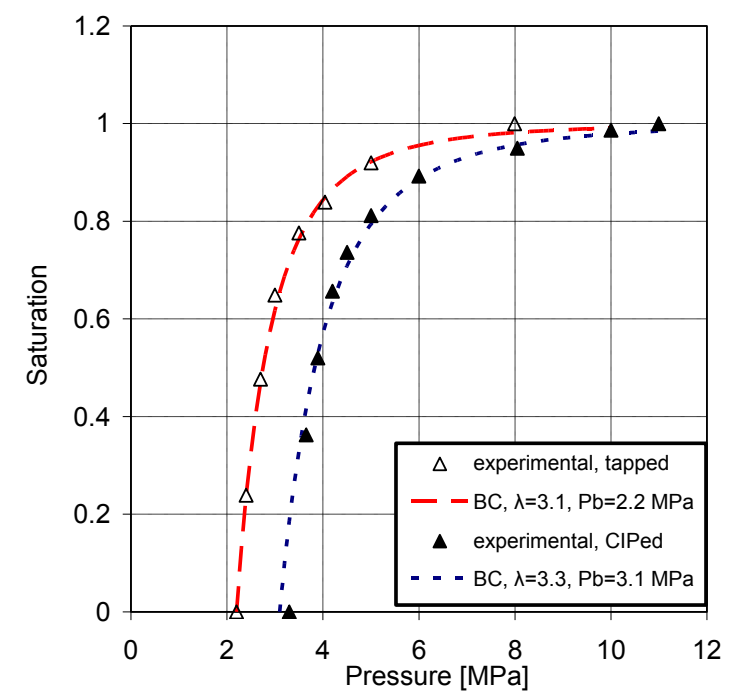

Fig. 5. Drainage curves for angular $\mathrm{Al}_{2} \mathrm{O}_{3}(\mathrm{~F} 1000) / \mathrm{Cu}$ a) with a preform made by tapping and vibrating $\left(V_{r}=0.48\right)$ at $1100{ }^{\circ} \mathrm{C}$ and $\left.\mathrm{b}\right)$ a preform made by cold isostatic pressing at $150 \mathrm{MPa}\left(V_{r}=0.53\right)$ and infiltrated at $1150{ }^{\circ} \mathrm{C}$. Each curve is fitted with the Brooks and Corey correlation (Eq. 7; see Fig. 7) for appropriate $P_{\mathrm{b}}$ and $\lambda$. $S=1$ was assumed at $8 \mathrm{MPa}$ for tapped preform and at $11 \mathrm{MPa}$ for CIPed preform.

\section{Discussion}

\section{A. Improvements to the technique}

The difference in precision between the old and the new set-up is clearly illustrated by the data in Fig. 3. With the new apparatus, precise and automatically controlled pressurization rates (here at $10^{-4} \mathrm{MPa} / \mathrm{s}$ and $\left.1.1 \cdot 10^{-3} \mathrm{MPa} / \mathrm{s}\right)$, as well as temperature $\left(1100^{\circ} \mathrm{C} \pm 4^{\circ} \mathrm{C}\right)$, remain constant during the whole infiltration process, lasting more than 3 hours. These improvements are brought about by the combination of an improved heating system, together with automated and finer pressure and temperature regulation systems. These make for a strongly improved capacity to compensate for two main factors: (i) ingress of pressurized cold gas into the chamber at high temperature produces a decrease of temperature, (ii) starting around 2-4 MPa gas pressure, heat losses by conduction and convection of heat through the gas to the cooled walls of the pressure infiltration apparatus become important.

Figure 4a compares data before (f.m.) and after (c.m.) modifications of the system. Good agreement is obtained, confirming that both methods can generate reliable drainage curves; however, improvements of the apparatus now allow to work in a much broader range of experimental conditions, including pressurization rates spanning the range from $10^{-1}$ to $10^{-4}$ $\mathrm{MPa} / \mathrm{s}$ while maintaining high temperatures stable within a few degrees $\left( \pm 5^{\circ} \mathrm{C}\right.$ at $\left.1300^{\circ} \mathrm{C}\right)$. Such slow infiltration under stable thermal conditions was not feasible with the older version of the apparatus.

\section{B. Pressurization rate for quasi-static infiltration under continuous pressurization}

Suitable computing parameters (in terms of step size $\left(\Delta x=2.5 \cdot 10^{-3} \mathrm{~m}\right)$ and stability $\left(\Delta t=10^{-5}\right.$ s)) for isothermal unidirectional infiltration under constant pressure $P_{0}$ were first identified, by comparison of predictions with those of a semi-analytical solution based on the similarity solution method, which holds for infiltration of semi-infinite media under constant pressure 
(calculated by Prof. V.J. Michaud, EPFL, as described in Refs. [17, 20]) with $k_{\mathrm{r}}$ from Eq. 9 or Eq. 10, Fig. 6. The good agreement validates the present finite-difference model, which was then used to simulate infiltration under variable increasing pressure.

The Brooks and Corey parameters put in the code are calculated from Fig. 5 for each of the two preform types. Replotting these data in the appropriate coordinates-log $(1-S)$ versus $\log (P)$ - should lead to a straight line if the Brooks and Corey relation for saturation is obeyed. One finds that Eq. 7 is indeed followed by the data, Fig. 7. The two parameters of the correlation $\left(P_{\mathrm{b}}\right.$ and $\lambda$ ) can then be deduced, respectively as $P_{\mathrm{b}}=2.2 \mathrm{MPa}$ with $\lambda=3.1 \pm 0.1$ for F1000 alumina preforms made by tapping and vibrating, and $P_{\mathrm{b}}=3.1 \mathrm{MPa}$ with $\lambda=3.3 \pm 0.1$ for F1000 alumina preforms made by CIP (uncertainties on $\lambda$ given above were evaluated according to Ref. [42]). That fact that $\lambda$ takes (within error) the same value for both preforms is expected, given that these were made of the same powder. The same effect was already observed in Ref. [18] and confirms that $\lambda$ reflects mostly the particle shape, as was initially proposed by Brooks and Corey [34] (we note in passing that a nominally similar F1000 alumina powder used in earlier experiments gave a slightly different $\lambda$ value in the range 2.6 to 2.9 [41]).

If one assumes that $P_{\mathrm{b}}$ scales with the work of immersion $[18,24]$, when $V_{\mathrm{r}}$ is increased from 0.48 to 0.53 the corresponding lines should be shifted horizontally by a factor of

$$
\frac{V_{r}^{C I P e d}}{V_{r}^{\text {tapped }}} \cdot \frac{1-V_{r}^{\text {tapped }}}{1-V_{r}^{C I P e d}}=\frac{0.53}{0.48} \cdot \frac{0.52}{0.47} \approx 1.2
$$

which is near, albeit somewhat lower than, the measured shift, which corresponds to a factor nearer 1.4. Cold-pressing having damaged some particles, the $50{ }^{\circ} \mathrm{C}$ difference in infiltration temperature plus uncertainty in the measurements might explain the discrepancy.

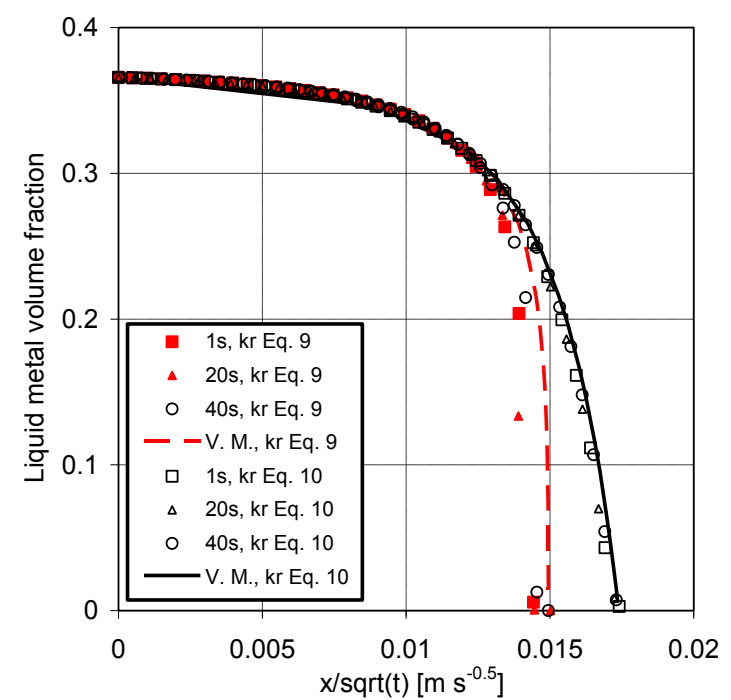

Fig. 6: Comparison of the semi-analytical similarity solution ("V.M.", calculated by Prof. V. Michaud of EPFL) and finite difference numerical simulation results for unidirectional infiltration according to the Brooks and Corey

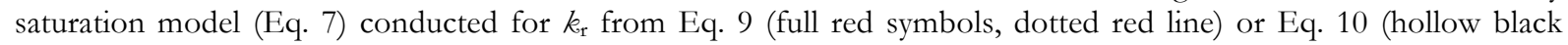
symbols, full black line), with a constant inlet pressure $P_{0}$ after 1,20 and $40 \mathrm{~s}\left(P_{0}=5 \mathrm{MPa}, \lambda=4.9, P_{\mathrm{b}}=2 \mathrm{MPa}, k_{\mathrm{s}}=\right.$ $\left.5 \cdot 10^{-14} \mathrm{~m}^{2}, \mu=3 \cdot 10^{-3} \mathrm{~Pa} \cdot \mathrm{s}, V_{\mathrm{r}}=0.63\right)$; abscissa is the similarity solution variable, namely distance divided by the square root of time. 


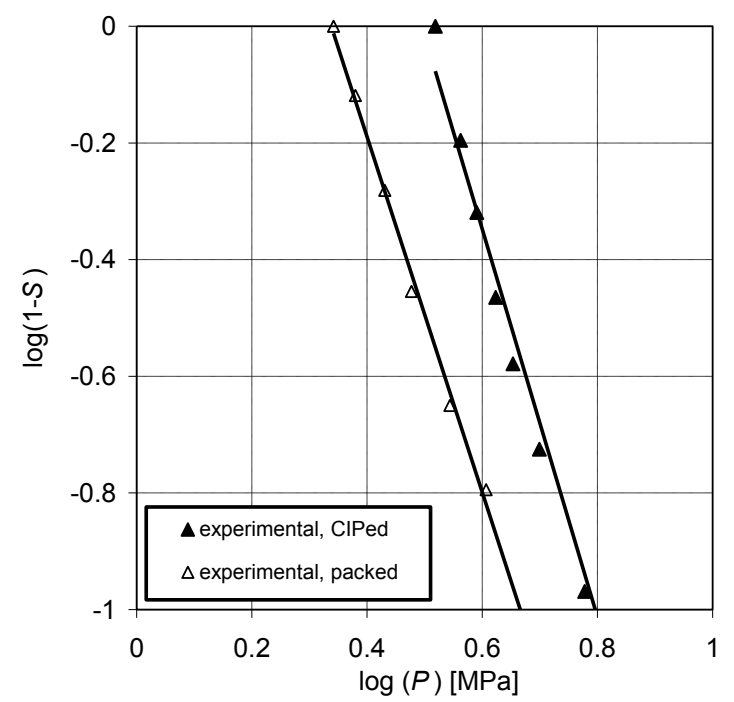

Fig. 7. Same curve as Fig. 5, replotted in terms of the Brooks-Corey correlation. $P_{\mathrm{b}}=2.2 \mathrm{MPa}$ and $\lambda=3.1$ for the preform made by tapping and vibrating and $P_{b}=3.1 \mathrm{MPa}$ and $\lambda=3.3$ for the preform made by CIP.

Simulations were then performed, using alternatively the relative permeability $k_{\mathrm{r}}$ given in Eq. 9 or that given in Eq. 10. Cold isostatically pressed preforms of Figs. 5 and 7 were considered; we assumed these to be infiltrated radially from the outer preform surface inwards with an outer preform diameter of $14 \mathrm{~mm}$, for each of the two pressurization rates of Fig. $4(0.04 \mathrm{MPa} / \mathrm{s}$ and $0.005 \mathrm{MPa} / \mathrm{s})$. Figure 8 illustrates the effect that the adopted expression for $k_{\mathrm{r}}$ has on the numerical predictions. This figure plots, for these two pressurization rates:

(i) the saturation profile at three different instants (Fig. 8a and 8b), and

(ii) the resulting curves of measured apparent saturation (i.e., the average saturation corresponding to the total volume of metal infiltrated into the preform at time $t$ ) versus pressure. Those curves are also compared with the Brooks-Corey saturation expression used in the calculation (Eq. 7 with parameters given above from Figs. 5 and 7), which one should obtain for quasi-static infiltration (Fig. 8c and 8d).
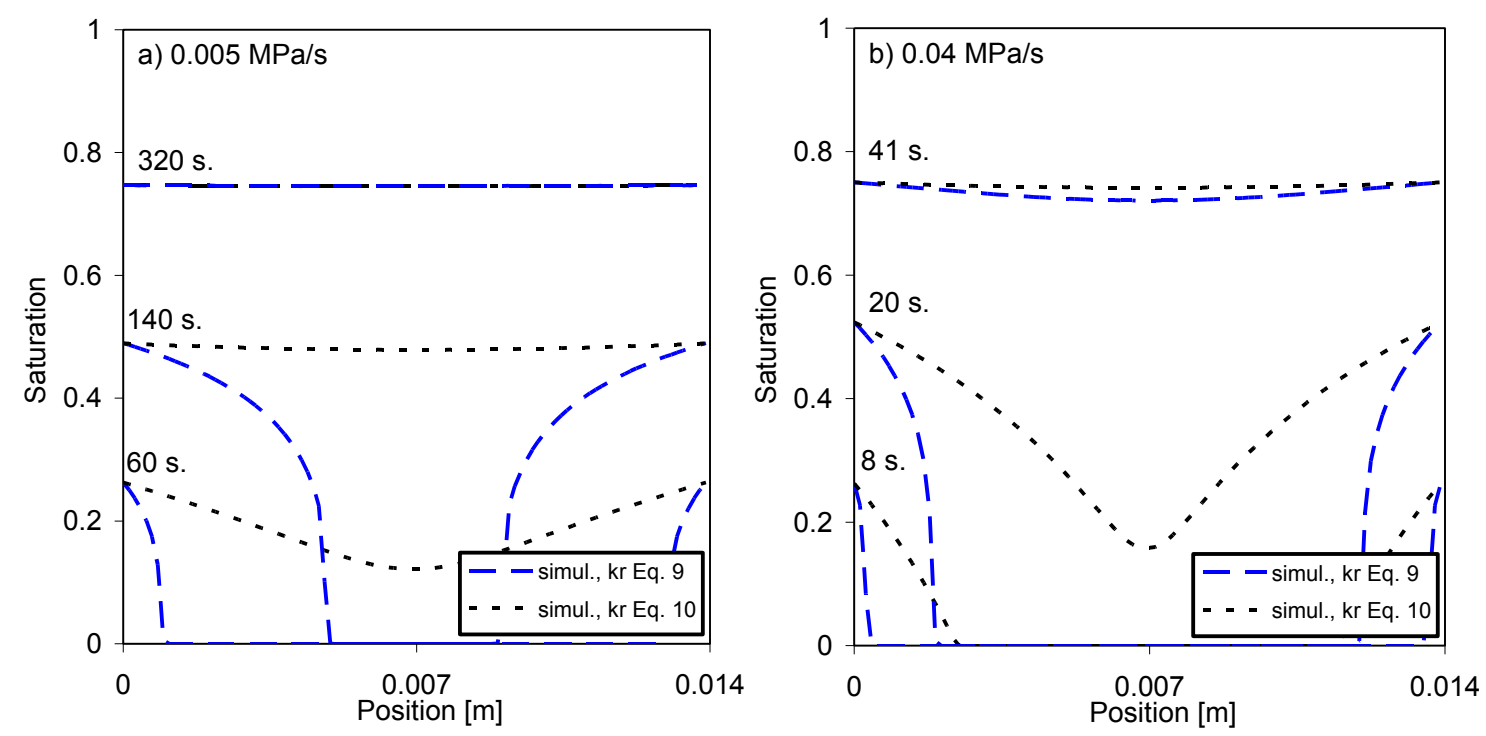

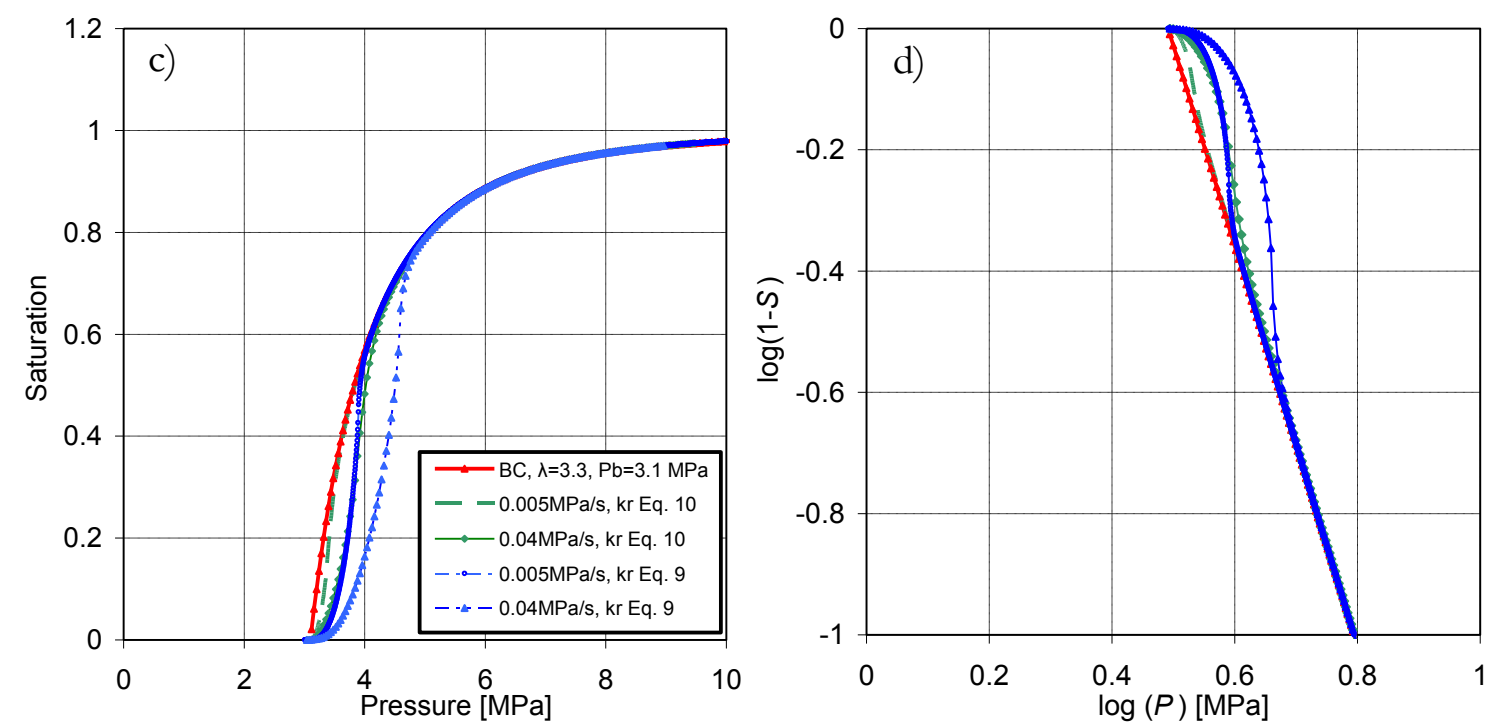

Fig. 8: Evolution of saturation across the preform depending on different conditions for relative permeability $k_{\mathrm{r}}$ given by Eq. 9 and Eq. 10. For a) $0.005 \mathrm{MPa} / \mathrm{s}$ after $60 \mathrm{~s}, 140 \mathrm{~s}$ and $320 \mathrm{~s}$ (starting from $P_{\mathrm{b}}$ ) and b) $0.04 \mathrm{MPa} / \mathrm{s}$ after $8 \mathrm{~s}$, $20 \mathrm{~s}$ and $41 \mathrm{~s}$. Corresponding drainage curves for c) two pressurization rates, namely $0.005 \mathrm{MPa} / \mathrm{s}$ and $0.04 \mathrm{MPa} / \mathrm{s}$ and d) same data plotted in $\log (1-S)$ vs $\log (P)$. The numerical parameters are: $\lambda=3.3, P_{\mathrm{b}}=3.1 \mathrm{MPa}, k_{\mathrm{s}}=0.42 \cdot 10^{-14}$ $\mathrm{m}^{2}, \mu=3.6 \cdot 10^{-3} \mathrm{~Pa} \cdot \mathrm{s}, V_{\mathrm{r}}=0.53$, calculated for radial infiltration of a cylinder of diameter $0.014 \mathrm{~m}$. "BC" stands for the Brooks and Corey saturation curve used in the simulation (Eq. 7 with $\lambda=3.3, P_{\mathrm{b}}=3.1 \mathrm{MPa}$ ).

As seen, the relative permeability value $k_{\mathrm{r}}$ exerts a strong influence on predicted infiltration kinetics, especially at lower pressure, i.e., near $P_{\mathrm{b}}$. Each drainage curve presents, indeed, a deviation (more and less accentuated) from the quasi-static Brooks and Corey saturation expression, Fig. 8c and 8d. Using $k_{\mathrm{r}}$ from Eq. 10 leads to the prediction that the saturation is essentially uniform across the preform at all times with a pressurization rate of $0.005 \mathrm{MPa} / \mathrm{s}$, which hence reproduces faithfully the assumed "real" saturation curve (red "BC" line in Figs. $8 \mathrm{c} \& \mathrm{~d})$. At $0.04 \mathrm{MPa} / \mathrm{s}$ the saturation takes longer to become uniform; still, the curve traces, at higher pressures, a significant portion of the "real" (Brooks and Corey) saturation curve.

As the relative permeability $k_{\mathrm{r}}$ defined from the Brooks and Corey correlation (Eq. 9) is lower than $k_{\mathrm{r}}$ from Eq. 10 for the same saturation and also decreases more rapidly with decreasing $S$ (Fig. 1), using this expression instead of Eq. 10 will predict much slower and less homogeneous infiltration. This is seen in Fig. 8, where strong gradients are predicted with this expression, and where a pressurization rate of $0.04 \mathrm{MPa} / \mathrm{s}$ largely underestimates the quasi-static drainage curve up to a saturation of 0.7. Experimental data on the other hand show no significant difference between drainage curves collected at $0.04 \mathrm{MPa} / \mathrm{s}$ or $0.005 \mathrm{MPa} / \mathrm{s}$, Fig. 4. This suggests that Eq. 9 is not suitable in the present copper/alumina system, while Eq. 10 seems to produce adequate predictions. In this we agree with the findings of Ref. [23], who proposed Eq. 10 by comparison of their simulation with data for aluminum infiltrating chopped alumina fibre preforms.

\section{Isobaric infiltration}

Calibrating the model with relevant values of $P_{\mathrm{b}}$ and $\lambda$ for the two preform types (Figs. 5 and 7), predictions of the unidirectional infiltration model can also be compared with data from the two infiltration experiments that produced the data in Fig. 5. Note that in the former case infiltration is assumed to take place from the top only; hence unidirectional infiltration of a finite slab was simulated, its thickness $L$ being taken as twice the preform height, namely $30 \mathrm{~mm}$ (in Cartesian coordinate system). With CIPped preforms on the other hand, since the preform is free-standing within the crucible, radial infiltration of the cylindrical preforms was assumed, the preform diameter being $14 \mathrm{~mm}$. Figure 9 compares the numerical (calculated with $k_{\mathrm{r}}$ from Eq. 9 or Eq. 10) and experimental saturation versus time for pressure ramps in these two stepwise infiltration experiments. 

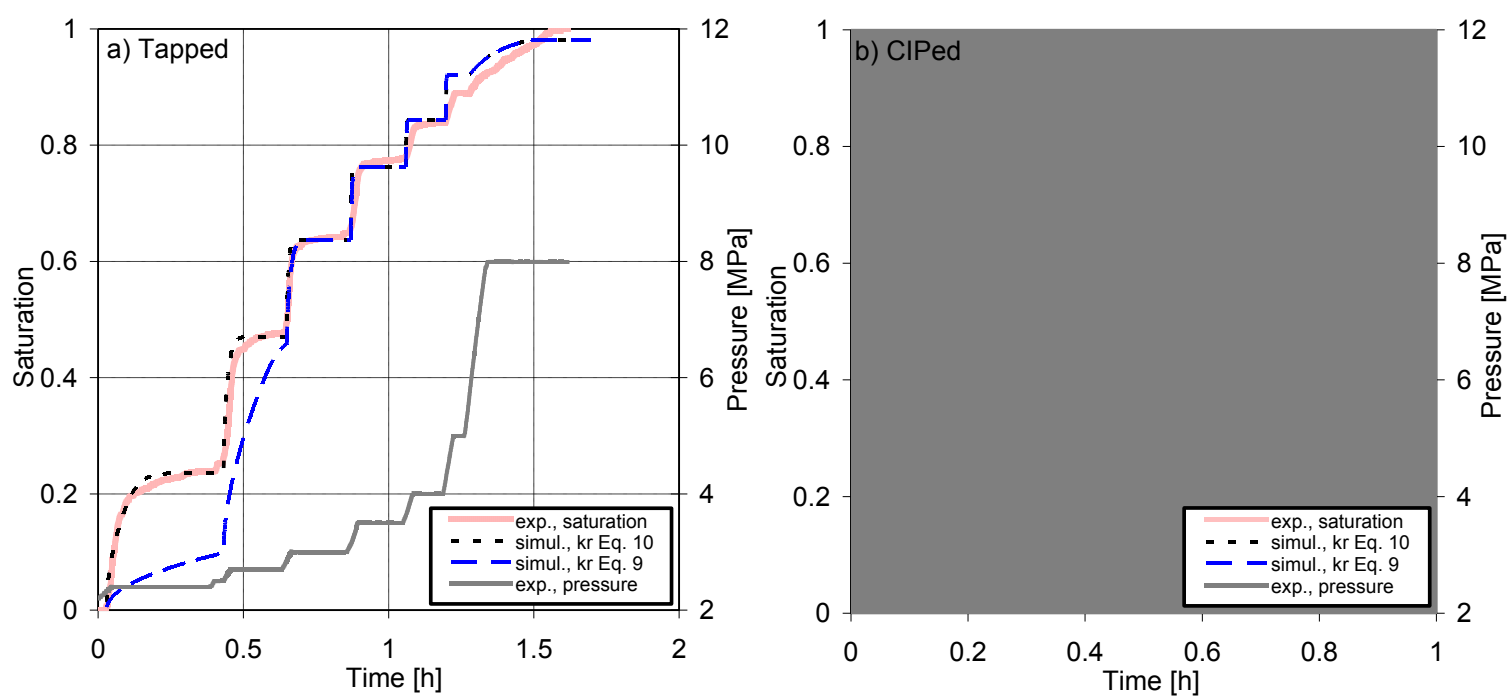

Fig. 9. Plot of pressure applied together with experimental and theoretically predicted curves of saturation as a function of time for a) preform made by packing and vibrating $\left(\lambda=3.1, P_{\mathrm{b}}=2.2 \mathrm{MPa}, k_{\mathrm{s}}=0.7 \cdot 10^{-14} \mathrm{~m}^{2}, \mu=4.3\right.$. $\left.10^{-3} \mathrm{~Pa} \mathrm{~s}, V_{\mathrm{r}}=0.48\right)$ and b) preform made by CIP $\left(\lambda=3.3, P_{\mathrm{b}}=3.1 \mathrm{MPa}, k_{\mathrm{s}}=0.42 \cdot 10^{-14} \mathrm{~m}^{2}, \mu=3.9 \cdot 10^{-3} \mathrm{~Pa} \mathrm{~s}, V_{\mathrm{r}}\right.$ $=0.53$. Infiltration temperature is (a) $1100{ }^{\circ} \mathrm{C}$ and (b) $1150{ }^{\circ} \mathrm{C}$.

As seen in Fig. 9a, best fit for packed preform is again obtained using $k_{\mathrm{r}}$ from Eq. 10 while with Eq. 9 ( $k_{\mathrm{r}}$ from the Brooks and Corey correlation), numerical results underpredict significantly the infiltration rate for $P$ values near $P_{\mathrm{b}}$ (lower part of the curves in Fig. 9a). Above $S$ $=0.5$, the infiltration kinetics for both numerical approaches are the same. Simulation of the CIPped preform, on the other hand, predicts much faster infiltration than is observed at low pressure, also when $k_{\mathrm{r}}$ is calculated according to Eq. 10. We have at present no simple explanation for the discrepancy, which we are currently investigating by conducting more experiments.

More work, both theoretical and experimental, is thus needed; however, it emerges from the present comparison that the Brooks and Corey expression for the relative permeability, Eq. 9, is an underestimate of the permeability of the present ceramic preforms to flowing (strongly nonwetting) metal. Comparatively, a relative permeability equal to saturation (Eq. 10) gives a better approximation. In this connection it is also worth noting that the Brooks and Corey correlation is likely to be unsuitable at low saturation for a different reason, namely the fact that capillarity and flow are, in the initial stages of infiltration, also governed by percolation [38, 43-44].

\section{Conclusion}

- Improvements to the experimental procedures enable the exploration of an increased range of experimental conditions, including steady pressurization rates in the range $10^{-1}-10^{-4} \mathrm{MPa} / \mathrm{s}$ while maintaining stable high temperatures $\left(1300^{\circ} \mathrm{C} \pm 5^{\circ} \mathrm{C}\right)$ and tracking the displacement of the metal level with a precision of around 40-50 $\mu \mathrm{m}$ (for a total plunger displacement of $6 \mathrm{~mm}$ ). With this apparatus, the entire drainage curve characterizing capillarity in high-temperature infiltration of porous preforms by molten metal can be obtained in one single experiment, using either continuous or stepwise pressurization. The technique gives stable results if pressurization rates at or below $0.04 \mathrm{MPa} / \mathrm{s}$ are used, suggesting in turn that such pressurization rates are in, or near, the range where quasistatic infiltration obtains. The present apparatus is essentially a hightemperature metal analogue of a mercury porosimeter; however its function is somewhat different, in that a porosimeter is generally used to measure a pore size distribution (based on the assumption of a constant value for the contact angle of $\mathrm{Hg}$ ), whereas the present apparatus is designed to measure wetting characteristics in various material systems (providing, thus, both capillary and geometric information). 
- Semi-empirical equations derived in the context of soil science for flow of two immiscible fluids in a porous medium are applied to pressure infiltration of ceramic preforms with liquid metal. A finite difference code for the isothermal unidirectional infiltration of a rigid preform under any steadily increasing pressure function is implemented. Numerical and experimental results show good agreement for the alumina-copper system for two different pressurization rates if one takes the relative permeability equal to the saturation, as was proposed in Ref. [23]; comparatively, the expression given by Brooks and Corey underestimates infiltration rates significantly. A discrepancy between numerical and experimental results for stepwise infiltration of cold isostatically pressed preforms is, however, noted; this is the subject of future work.

\section{Acknowledgments}

This work is sponsored by the Swiss National Science Foundation, Project No. 200020137685. The authors wish to express their gratitude to Prof. Véronique Michaud and Dr. Alain Jacot, both of EPFL, for their assistance and advice concerning the simulation part of this work. 


\section{References}

1. N. Eustathopoulos and A. Mortensen, in: Fundamentals of Metal Matrix Composites, S. Suresh, A. Mortensen, A. Needleman (Eds.), pp. 42-58. Butterworth-Heinemann, Boston (1993).

2. A. Mortensen and I. Jin, Solidification Processing of Metal Matrix Composites. International Materials Reviews 37, 101 (1992).

3. V. J. Michaud, in: Fundamentals of Metal Matrix Composites, S. Suresh, A. Mortensen, A. Needleman (Eds.), pp. 3-22. Butterworth-Heinemann, Stoneham, Mass (1993).

4. D. J. Lloyd, A. D. Morris and I. Jin, US Patent No. 5,028,392 (1991).

5. A. Mortensen, in: Comprehensive Composite Materials : Metal Matrix Composites, T. W. Clyne (Ed.), Vol. 3, pp. 521-554. Pergamon, Oxford UK (2000).

6. R. Asthana, Solidification Processing of Reinforced Metals. Trans-Tech Publications Ltd., Uetikon-Zurich (1998).

7. A. Evans, C. SanMarchi and A. Mortensen, Metal Matrix Composites in Industry: an Introduction and a Survey. Kluwer Academic Publishers, Dordrecht NL (2003).

8. V. Michaud and A. Mortensen, Infiltration Processing of Fibre Reinforced Composites: Governing Phenomena. Composites: Part A, Applied Science and Manufacturing 32, 981 (2001).

9. C. Garcia-Cordovilla, E. Louis and J. Narciso, Pressure Infiltration of Packed Ceramic Particulates by Liquid Metals. Acta materialia 47, 4461 (1999).

10. N. Chawla and K. K. Chawla, Metal Matrix Composites. Springer Verlag, New York (2006).

11. A. W. Adamson, Physical Chemistry of Surfaces, 4th Edition. J. Wiley \& Sons, New York (1982).

12. N. Eustathopoulos, M. G. Nicholas and B. Drevet, Wettability at High Temperature. Pergamon-Elsevier Science, Amsterdam NL (1999).

13. P. G. deGennes, F. Brochard-Wyart and D. Quéré, Capillarity and Wetting Phenomena Drops, Bubbles, Pearls, W aves. Springer Science+Business Media, New York, USA (2004).

14. N. Eustathopoulos, N. Sobczak, A. Passerone and K. Nogi, Measurement of contact angle and work of adhesion at high temperature. Journal of Materials Science 40, 2271 (2005).

15. N. R. Morrow, Physics and Thermodynamics of Capillary Action in Porous Media. Industrial and Engineering Chemistry 62, 32 (1970).

16. J. Bear, Dynamics of Fluids in Porous Media. American Elsevier, New York (1972).

17. V. J. Michaud, L. Compton and A. Mortensen, Capillarity in Isothermal Infiltration of Alumina Fiber Preforms with Aluminum. Metallurgical Transactions 25A, 2145 (1994).

18. M. Bahraini, L. Weber, J. Narciso and A. Mortensen, Wetting in infiltration of alumina particle preforms with molten copper. Journal of Materials Science 40, 2487 (2005).

19. J. M. Molina, J. Narciso and E. Louis, On the triple line in infiltration of liquid metals into porous preforms. Scripta Materialia 62, 961 (2010).

20. V. Michaud and A. Mortensen, On measuring wettability in infiltration processing. Scripta Materialia 56, 859 (2007).

21. A. Mortensen, Interfacial Phenomena in the Solidification Processing of Metal Matrix Composites. Materials Science and Engineering A135, 1 (1991).

22. H. Kaufmann and A. Mortensen, Wetting of Saffil ${ }^{\mathrm{TM}}$ Alumina Fiber Preforms by Aluminum at 973 K. Metallurgical Transactions 23A, 2071 (1992).

23. T. Dopler, A. Modaressi and V. J. Michaud, Simulation of Metal Composite Isothermal Infiltration Processing. Metallurgical and Materials Transactions 31B, 225 (2000).

24. M. Bahraini et al., Measuring and tailoring capillary forces during liquid metal infiltration. Current Opinion in Solid State and Materials Science 9, 196 (2005).

25. M. Bahraini, J. M. Molina, L. Weber and A. Mortensen, Direct measurement of drainage curves in infiltration of SiC particle preforms. Materials Science and Engineering: A 495, 203 (2008). 
26. M. Kida, M. Bahraini, J. M. Molina, L. Weber and A. Mortensen, High-temperature wettability of aluminum nitride during liquid metal infiltration. Materials Science and Engineering: A 495, 197 (2008).

27. F. A. L. Dullien, Porous Media, Fluid Transport and Pore Structure. Academic Press, New York (1979).

28. J. Bear and Y. Bachmat, Introduction to Modeling of Transport Phenomena in Porous Media. Kluwer Academic Publishers, Dordrecht, The Netherlands (1990).

29. A. Rodriguez, S. Sanchez, J. Narciso, E. Louis and F. Rodriguez-Reinoso, Pressure infiltration of $\mathrm{Al}-\mathrm{Si}$ alloys into compacts made of carbon particles. Journal of Materials Science 40, 2519 (2005).

30. J. Molina et al., Threshold pressure for infiltration and particle specific surface area of particle compacts with bimodal particle distributions. Scripta Materialia 51, 623 (2004).

31. M. Kida, M. Bahraini, J. M. Molina, L. Weber and A. Mortensen, High-temperature wettability of aluminum nitride during liquid metal infiltration. Materials Science and Engineering: A 495, 197 (2008).

32. J. M. Molina, R. Prieto, M. Duarte, J. Narciso and E. Louis, On the estimation of threshold pressures in infiltration of liquid metals into particle preforms. Scripta Materialia 59, 243 (2008).

33. J. M. Molina et al., Infiltration of graphite preforms with Al-Si eutectic alloy and mercury. Scripta Materialia 56, 991 (2007).

34. R. H. Brooks and A. T. Corey, Hydraulic Properties of Porous Media, Colorado State University Hydrology Papers, (1964).

35. V. J. Michaud, in: Composite reinforcements for optimum performance, P. Boisse (Ed.), pp. 431458. Woodhead Publishing, Oxford UK (2011).

36. A. Mortensen, L. J. Masur, J. A. Cornie and M. C. Flemings, Infiltration of Fibrous Preforms by a Pure Metal: Part I. Theory. Metallurgical Transactions 20A, 2535 (1989).

37. Y. Mualem, A new model for predicting the hydraulic conductivity of unsaturated porous media. Water Resour. Res. 12, 513 (1976).

38. A. Hunt and R. Ewing, Percolation Theory for Flow in Porous Media. Springer, Heidelberg, Germany (2009).

39. D. R. Poirier and G. H. Geiger, Transport Phenomena in Materials Processing. TMS, Warrendale, PA (1994).

40. W. F. Gale and T. C. Totemeier, Smithells Metals Reference Book (8th Edition). Elsevier (2004).

41. M. Bahraini, Characterization of capillary forces during liquid metal infiltration, $\mathrm{PhD}$ thesis, Laboratory for Mechanical Metallurgy, Institute of Materials, Ecole Polytechnique Fédérale de Lausanne, Lausanne (2007).

42. J. Higbie, Uncertainty in the linear regression slope. Am. J. Phys. 59, 184 (1991).

43. M. Sahimi, Flow phenomena in rocks: from continuum models to fractals, percolation, cellular automata, and simulated annealing. Reviews of Modern Physics 65, 1393 (1993).

44. J. F. Gouyet, Physics and Fractal Structures. Masson, Paris and Springer, New York (1996). 